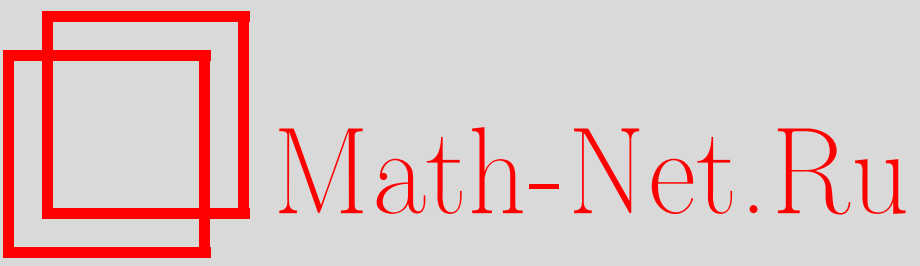

Л. Д. Пустыльников, Обобщенные цепные дроби, связанные с преобразованием Гаусса, УМН, 2002, том 57, выпуск 5, 159-160

DOI: https://doi.org/10.4213/rm564

Использование Общероссийского математического портала Math-Net.Ru подразумевает, что вы прочитали и согласны с пользовательским соглашением

http://www.mathnet.ru/rus/agreement

Параметры загрузки:

IP : 54.209 .52 .79

26 апреля 2023 г., 03:38:03 


\title{
ОБОБЩЕННЫЕ ЦЕПНЫЕ ДРОБИ, СВЯЗАННЫЕ С ПРЕОБРАЗОВАНИЕМ ГАУССА
}

\author{
Л. Д. Пустыльников
}

Общая конструкция разложения произвольного $n$-мерного вектора $x$ в обобщенную $(A, \omega)$ цепную дробь $x=\left[q^{(0)}, q^{(1)}, \ldots\right]_{A, \omega}$, предложенная в $[1],[2]$, зависит от некоторого преобразования $A n$-мерного линейного пространства и $n$-мерного вектора $\omega$, принадлежащего $n$-мерному единичному тору $T_{n}=\left\{y_{1}, \ldots, y_{n}: 0 \leqslant y_{s}<1 ; s=1, \ldots, n\right\}$. Если же $n=1, \omega=0$, а отображение $A$ имеет вид $A=\Gamma: x \rightarrow 1 / x$ и проектируется на единичную окружность $T_{1}$ в виде отображения $Г$ аусса $\bar{\Gamma}: y \rightarrow 1 / y \bmod 1$, то $(A, \omega)$-цепная дробь совпадает с обычной цепной дробью $x=\left[a_{0}, a_{1}, \ldots\right]$ [3], где $a_{k}$ - неполные частные. В данной работе мы изучаем частный случай общего понятия $(A, \omega)$-цепной дроби, когда $n=1, x$ - число, $A=\Gamma$, а число $\omega$ удовлетворяет неравенству $0 \leqslant \omega<1$. Такую цепную дробь в случае, если она конечная, мы будем обозначать $x=\left[a_{0}, \ldots, a_{m}\right]_{\omega}$ и назьвать конечной $\omega$-цепной дробью, а в случае, если эта дробь бесконечная, мы будем обозначать ее $x=\left[a_{0}, a_{1}, \ldots\right] \omega$ и назьвать бесконечной $\omega$-цепной дробью. Разложения в $\omega$-цепную дробь мы будем применять только для иррациональных чисел $x$. Опишем формалшное построение $\omega$-цепных дробей. Пусть $x$ - иррациональное число, а $\omega$ - произвольное число, удовлетворяющее неравенству $0 \leqslant \omega<1$.

Пусть $a_{0}=[x]$ - целая часть $x$. Полагаем $x=x_{0}=a_{0}+\delta_{0}$. Если $\delta_{0}=\omega$, то процесс построения $\omega$-цепной дроби числа $x$ на этом заканчивается, эта дробь конечная и $x=\left[a_{0}\right]_{\omega}$. Если же $\delta_{0} \neq \omega$, то в силу того, что число $x$ - иррациональное, имеем неравенство $\delta_{0} \neq 0$ и полагаем $x_{1}=1 / \delta_{0}$. Предположим теперь, что при некотором целом $k \geqslant 0$ построены целше числа $a_{0}, a_{1}, \ldots, a_{k}$ и числа $x_{0}, \ldots, x_{k+1}$ такие, что $x_{s}=a_{s}+\delta_{s}$ и $\delta_{s} \neq \omega$ при $s=0, \ldots, k$. Пусть $a_{k+1}=\left[x_{k+1}\right]$, и полагаем $x_{k+1}=a_{k+1}+\delta_{k+1}$. Если $\delta_{k+1}=\omega$, то процесс построения $\omega$-цепной дроби числа $x$ заканчивается, эта дробь конечная и $x=\left[a_{0}, \ldots, a_{k+1}\right]_{\omega}$. Если же $\delta_{k+1} \neq \omega$, то в силу того, что число $x$ - иррациональное, $\delta_{k+1} \neq 0$, и поэтому полагаем $x_{k+2}=1 / \delta_{k+1}$. Если при всех целых $k$ выполнено неравенство $\delta_{k} \neq \omega$, то полагаем $x=\left[a_{0}, a_{1}, \ldots\right] \omega$ и $\omega$-цепная дробь числа $x$ - бесконечная. Представление $x$ в виде $\omega$-цепной дроби описано.

ЗАмечАниЕ. Если $\omega=0$, то $\omega$-цепная дробь совпадает с обычной цепной дробью.

ОПРЕДЕлЕНиЕ 1. Для любой бесконечной $\omega$-цепной дроби $x=\left[a_{0}, a_{1}, \ldots\right] \omega$ числа $x$ и любого целого числа $n \geqslant 0$ определим $\omega$-подходящую дробь $\mathscr{S}_{\omega}^{(n)}$ следующим образом: $\mathscr{S}_{\omega}^{(n)}=$ $\left[a_{0}, \ldots, a_{n}\right]_{\omega}$.

Далее будем обозначать подходящую дробь для обычной цепной дроби числа $x$ в виде $p_{n} / q_{n}=$ $\left[a_{0}, \ldots, a_{n}\right]$, где $p_{n}, q_{n}(n \geqslant 0)$ - целые числа, связанные между собой с помощью равенств $p_{n}=p_{n-1} a_{n}+p_{n-2}, q_{n}=q_{n-1} a_{n}+q_{n-2}$, где $p_{-1}=1, q_{-1}=0$ и $p_{0}=[x], q_{0}=1$.

ЛЕмма. Пусть $\mathscr{S}_{\omega}^{(n)}=\left[a_{0}, \ldots, a_{n}\right]_{\omega}-$ конечная $\omega$-цепная дробь, а $p_{k} / q_{k}=\left[a_{0}, \ldots, a_{k}\right]$ $(k \leqslant n)-$ обычные конечные цепные дроби. Тогда при $n \geqslant 2$ выполнено равенство $\mathscr{S}_{\omega}^{(n)}=p_{\omega}^{(n)} / q_{\omega}^{(n)}$, где $p_{\omega}^{(n)}=p_{n}+p_{n-1} \omega, q_{\omega}^{(n)}=q_{n}+q_{n-1} \omega$.

ТЕорема 1. Два различных иррациональных числа имеют различные разложения в $\omega$-цепную дробь.

Teоpema 2. Пусть $n \geqslant 1, \beta=\left(1-\omega^{2}\right) / \omega$. Тогда:

1) если $a_{n}>\beta$, то знак $\operatorname{sign}\left(\mathscr{S}_{\omega}^{(n)}-\mathscr{S}_{\omega}^{(n-1)}\right)=(-1)^{n}$, а если $a_{n}<\beta$, то знак $\operatorname{sign}\left(\mathscr{S}_{\omega}^{(n)}-\mathscr{S}_{\omega}^{(n-1)}\right)=(-1)^{n+1}$

2) вьполняется неравенство $\left|\mathscr{S}_{\omega}^{(n)}-\mathscr{S}_{\omega}^{(n-1)}\right| \leqslant\left|a_{n}-\beta\right| \omega /\left(q_{\omega}^{(n)} q_{\omega}^{(n-1)}\right)$.

TEOPEMA 3. Пусть $n \geqslant 2, \gamma=\omega a_{n} a_{n-1}+\omega^{2} a_{n-1}$. Ecли $a_{n}>\gamma, \operatorname{mo~знак~} \operatorname{sign}\left(\mathscr{S}_{\omega}^{(n)}-\right.$ $\left.\mathscr{S}_{\omega}^{(n-2)}\right)=(-1)^{n}$, а если $a_{n}<\gamma$, то знак $\operatorname{sign}\left(\mathscr{S}_{\omega}^{(n)}-\mathscr{S}_{\omega}^{(n-2)}\right)=(-1)^{n-1}$. 
Tеорема 4. Пусть $x=\left[a_{0}, a_{1}, \ldots\right] \omega$. Тогда при $n \geqslant 2$ имеют место следующие равенства:

$$
\begin{aligned}
& p_{\omega}^{(n)}=a_{n} p_{\omega}^{(n-1)}+p_{\omega}^{(n-2)}+\left(a_{n}-a_{n-1}\right) \sum_{k=-1}^{n-2}(-\omega)^{n-k-1} p_{\omega}^{(k)}, \\
& q_{\omega}^{(n)}=a_{n} q_{\omega}^{(n-1)}+q_{\omega}^{(n-2)}+\left(a_{n}-a_{n-1}\right) \sum_{k=0}^{n-2}(-\omega)^{n-k-1} q_{\omega}^{(k)},
\end{aligned}
$$

əде $p_{\omega}^{(-1)}=1$.

СлЕДСТВиЕ 1. При всех $\omega$, для которых выполнено равенство $a_{n}=a_{n-1}$, соотношения, вьражающие числитель и знаменатель $\omega$-подходящей дроби $\mathscr{S}_{\omega}^{(n)}$ через числители и знаменатели $\omega$-подходящих дробей $\mathscr{S}_{\omega}^{(k)}$ nри $k<n$, совпадают с классическими соотношениями.

ОПРЕДЕЛЕниЕ 2 . Если $x=\left[a_{0}, \ldots, a_{n}\right]_{\omega}$ - конечная $\omega$-цепная дробь, а $s$-натуральное число, удовлетворяющее условию $1 \leqslant s \leqslant n$, то $\omega$-остаток $r_{\omega}^{(s)}$ есть конечная $\omega$-цепная дробь $r_{\omega}^{(s)}=\left[a_{s}, \ldots, a_{n}\right]_{\omega}$, а если $x=\left[a_{0}, a_{1}, \ldots\right]_{\omega}-$ бесконечная $\omega$-цепная дробь, то $\omega$-остаток $r_{\omega}^{(s)}$ есть бесконечная $\omega$-цепная дробь $r_{\omega}^{(s)}=\left[a_{s}, a_{s+1}, \ldots\right] \omega$.

Теорема 5 . Пусть $1 \leqslant s \leqslant n$. Тогда справедливо равенство

$$
\left[a_{0}, \ldots, a_{n}\right]_{\omega}=\frac{p_{\omega}^{(s-1)} r_{\omega}^{(s)}+\left(1-\omega r_{\omega}^{(s)}\right) \sum_{k=-1}^{s-2}(-\omega)^{s-2-k} p_{\omega}^{(k)}}{q_{\omega}^{(s-1)} r_{\omega}^{(s)}+\left(1-\omega r_{\omega}^{(s)}\right) \sum_{k=0}^{s-2}(-\omega)^{s-2-k} q_{\omega}^{(k)}}
$$

где $p_{\omega}^{(-1)}=1, p_{\omega}^{(k)} / q_{\omega}^{(k)}=\left[a_{0}, \ldots, a_{k}\right]_{\omega}, r_{\omega}^{(s)}=\left[a_{s}, a_{s+1}, \ldots, a_{n}\right]_{\omega}$.

ТЕОРема 6. Иррациональное число х является квадратичной иррациональностью тогда и только тогда, когда значения $\omega$ на полуинтервале $0 \leqslant \omega<1$, для которьх $\omega$-цепная дробь числа $x$ - конечная, образуют конечное множество.

ОПредЕЛЕниЕ 3 . Для данного иррационального числа $x$ введем множество $\Omega_{x}$ на полуинтервале $[0,1)$, состоящее из всех таких чисел $\omega \in[0,1)$, для которых $\omega$-цепная дробь числа $x-$ конечная.

СлЕДСТВИЕ 2. Если число $х$ не является квадратичной иррациональностью, то множество $\Omega_{x}$ - счетное и состоит из попарно различных чисел, обратных всем остаткам в разложсении числа $x$ в обычную чепную дробь.

Будем считать $\Omega_{x}$ упорядоченной последовательностью, а его элементы обозначать через $\omega_{n}^{(x)}=1 / r_{n}(n=0,1, \ldots)$, где $x=\left[a_{0}, a_{1}, \ldots\right], r_{n}=\left[a_{n}, a_{n+1}, \ldots\right]$.

Теорема 7. Число $x$ есть алгебраическое число степени $n$ тогда и только тогда, когда множество $\Omega_{x}$ состоит только из алгебраических чисел степени $n$, принадлежащих расширению поля рациональных чисел, полученного присоединением числа $x$.

\section{СПИСОК ЛИТЕРАТУРЫ}

[1] Л. Д. Пустыльников // Матем. заметки. 1994. Т. 56. №6. C. 144-148. [2] L. D. Pustyl'nikov // J. Math. Sci. 1999. V. 95. № 5. Р. 2552-2563. [3] А. Я. Хинчин. Цепные дроби. М.: Физматлит, 1961. 